\title{
Functionalization of Graphene and Dielectric Property Relationships in PVDF/graphene Nanosheets Composites
}

\author{
Xuewen Zheng ${ }^{1}$, Huitao $Y u^{1}$, Shuangshuang Yue ${ }^{1}$, Ruiguang Xing ${ }^{1}$, Qiwei Zhang ${ }^{1 *}$, Yunying Liu ${ }^{2}$, \\ Bangwen Zhang ${ }^{1}$ \\ ${ }^{1}$ Inner Mongolia Key Laboratory of Ferroelectric New Energy Materials \& Devices, School of \\ Materials and Metallurgy, Inner Mongolia University of Science and Technology, 7\# Arding Street, \\ Kun District, Baotou 014010, China \\ ${ }^{2}$ School of Chemistry and Chemical Engineering, Inner Mongolia University of Science and \\ Technology, 7\# Arding Street, Kun District, Baotou 014010, China \\ *E-mail: zqw8000@imust.edu.cn
}

doi: $10.20964 / 2018.01 .16$

Received: 29 August 2017 / Accepted: 25 October 2017 / Online Published: 1 December 2017

In this paper, a series of the functionalized graphenes, such as graphene oxide (GO), reduced graphene oxide (rGO), carboxylated graphene oxide $(\mathrm{GOCOOH})$, reduce carboxylated graphene oxide $(\mathrm{rGOCOOH})$, fluorinated graphene oxide $(\mathrm{GOF})$, and reduce fluorinated graphene oxide (rGOF), were successfully fabricated, and were introduced into the Poly (vinylidene fluoride) (PVDF) matrix to form composites. The effects of these functional groups on dielectric properties of GO/PVDF composites were systematically investigated. The results show that the reduced functional graphene/PVDF composites (rGOCOOH/PVDF, rGOF/PVDF) exhibit more excellent dielectric properties than that of composite samples without undergoing the reduction reaction (GOCOOH/PVDF, GOF/PVDF). The reduced samples not only possess high dielectric constant, but also maintain lower dielectric loss. And, the $\mathrm{rGOCOOH} / \mathrm{PVDF}$ composite has better dielectric properties $\left(\varepsilon_{\mathrm{r}}=17.64, \operatorname{tg} \delta=0.031\right)$ than others, due to strong interfacial and dipolar interactions between PVDF matrix and fillers. These results could be used as a guide to tune dielectric properties of polymer/ graphene composites for their applications in embedded capacitors.

Keywords: Functionalized graphenes; PVDF; Dielectric properties

\section{$\underline{\text { FULL TEXT }}$}

(C) 2018 The Authors. Published by ESG (www.electrochemsci.org). This article is an open access article distributed under the terms and conditions of the Creative Commons Attribution license (http://creativecommons.org/licenses/by/4.0/). 